\title{
Enabling an Online Community for Sharing Oral Medicine Cases Using Semantic Web Technologies*
}

\author{
Marie Gustafsson $^{1,2}$, Göran Falkman ${ }^{1}$, Fredrik Lindahl ${ }^{2}$, and Olof Torgersson ${ }^{2}$ \\ ${ }^{1}$ School of Humanities and Informatics, University of Skövde, \\ PO Box 408, SE-541 28 Skövde, Sweden \\ \{marie.gustafsson, goran.falkman\}@his.se \\ ${ }^{2}$ Computer Science and Engineering, Chalmers University of Technology \\ SE-412 96 Göteborg, Sweden \\ \{lindahlf, oloft\}@cs.chalmers.se
}

\begin{abstract}
This paper describes how Semantic Web technologies have been used in an online community for knowledge sharing between clinicians in oral medicine in Sweden. The main purpose of this community is to serve as repository of interesting and difficult cases, and as a support for monthly teleconferences. All information regarding users, meetings, news, and cases is stored in RDF. The community was built using the Struts framework and Jena was used for interacting with RDF.
\end{abstract}

\section{Introduction}

For a community to learn, ongoing learning by its members is vital. One means of supporting the transfer of individual knowledge into community knowledge is using computer supported tools, which can aid in making the knowledge communicable, consensual, and integrated into the community [1 2]. Further, tools supporting remote collaboration and consultation are important to all specialties of medicine 34, but especially for smaller fields, such as oral medicine, where specialists may be geographically dispersed.

The members of the Swedish Oral Medicine Network (SOMNet) are clinicians located at about ten different care-giving facilities throughout Sweden. They hold monthly teleconferences to discuss difficult and interesting cases. These remote meetings are currently supported by PowerPoint presentations. The clinicians have identified a need for a more structured online bank of cases covered at meetings, to serve as a collective memory for the group, and to provide better support for follow-ups of the discussed cases. It is believed that in creating, maintaining, and using this bank of cases, community learning will be enhanced.

The building of an online community for SOMNet is part of the SOMWeb (Swedish Oral Medicine Web) project. The project aims to obtain further knowledge about how interactive, user-centred knowledge-based systems supporting

\footnotetext{
* The work presented in this paper was supported by the Swedish Agency for Innovation Systems.
} 
evidence-based oral medicine should be designed, implemented and introduced in the daily clinical work [5]. Work on achieving the above aim is divided into three partially overlapping objectives: (1) The formalization of clinical processes and knowledge, (2) the development of web services for oral medicine, and (3) the construction of an intelligent web community.

To further frame the work of this online community, SOMWeb is in turn based on a medical information system called MedView, which contains the basis of SOMWeb in the form of elaborated content when it comes to services for knowledge management, together with an established user community [6]. To provide rapid prototyping and deployment of applications in daily clinical work, and to support the harmonization of clinical processes and knowledge within oral medicine, MedView has been developed in collaboration between experts within SOMNet and experts in computer science.

This paper describes an online community supporting processes of sharing and discussing cases in oral medicine. Previous work on objective one above, the formalization of clinical processes and knowledge in oral medicine [7], has described how Semantic Web technologies can be used to describe and encode medical examinations in oral medicine. This representation of cases will be underlying to the examinations stored in the online community. Semantic Web languages will also be used to describe other parts of the community, such as users, meetings, and news. A general description of the SOMWeb community, with no focus on the use of Semantic Web technology, has been shown as a software demonstration [8].

We begin by giving some background on use of Semantic Web technologies in the life sciences and in online communities. After a description of the current forms of collaboration in SOMNet and a survey of the clinicians desires of a more advanced system, we will present design choices we have made in constructing the SOMWeb community. The implementation of the community is described and some initial results reported. This is followed a short discussion, conclusions, and future work.

\section{Semantic Web Technologies}

This section will cover arguments for using Semantic Web technologies in medical informatics and in online communities. By Semantic Web technologies we refer foremost to using ontologies and the Web Ontology Language1 (OWL), describing data using Resource Description Framework 2 (RDF), and using SPARQL 3 as a query language and data access protocol for RDF data. Connected with these are other Semantic Web technologies, such as use of inference and rules.

\subsection{Semantic Web and the Life Sciences}

The life sciences are often mentioned as an area where Semantic Web technologies could give great improvements. Indeed, the W3C has an interest group devoted

\footnotetext{
1 http://www.w3.org/2004/OWL

2 http://www.w3.org/RDF

3 http://www.w3.org/TR/rdf-sparql-query/
} 
to the subject4. Areas where Semantic Web technologies can be applied include integration of heterogeneous data, locating relevant data sources and tools, retrieving relevant information, using inference to gather new insights, sharing formal annotations, creating rich and well-defined models of biological systems, and embedding models and semantics within online publications 9[10].

\subsection{Semantic Web and Online Communities}

A web portal "collects information for a group of users that have common interests" [11], and using Semantic Web technologies can help portals increase the consistency of information and quality of information processing [12]. In relation to online communities and web portals it is also relevant to mention Content Management System (CMS), which is software for facilitating collaborative creation and organization of documents and other content.

When describing people using Semantic Web technologies, the Friend of a Friend (FOAF) vocabulary 5 is often used. FOAF as a project aims to create a Web of machine-readable homepages "describing people, the links between them and the things they create and do." Indeed, several initiatives for online Semantic Web communities use FOAF in one way or another.

Semantic Web Advanced Development for Europe (SWAD-Europe) presents how Semantic Web tools and standards can be used to build a decentralized information portal [13, as used in the Semantic Web Environmental Directory (SWED) demonstrator. Each of the environmental organizations that want to be in the directory provides RDF descriptions of their organizations, constructed using a web-based data entry tool. The data is then hosted on the organization's own web site (similar to FOAF).

Another approach to adding Semantic Web technology support for communities has been to enable the sharing of forum posts and such between different communities, by defining a common format for these posts, as is done in the Semantically Interlinked Online Communities (SIOC) [14. Its goal is to interconnect online communities in helping to locate relevant and related information. The ontology and interface will let users searching in one forum find information on forums from other sites using a SIOC-based system architecture. The SIOC ontology outlines main classes, such as Site, Forum, Post, Event, Group, and User and properties of these. Mappings to e.g., FOAF are also provided.

SEmantic portAL (SEAL) [15] uses ontologies for dealing with the requirements of typical community web sites. Such requirements are information integration and web site management. The system architecture of SEAL includes a knowledge warehouse and the Ontobroker [16] system for inferencing. Community users and software agents access the Ontobroker through modules for navigation, query, templates for adding data, and an RDF generator.

The Semantic Web Research Community (SWRC) ontology [17] is an ontology for representing knowledge about researchers and research communities.

\footnotetext{
${ }^{4}$ http://www.w3.org/2001/sw/hcls/

5 http://www.foaf-project.org/
} 
Main concepts of this ontology are, among others, Organization, Project, Person, Topic, Publication, and Event. The SWRC ontology is used and extended by several portals based on the SEAL approach.

\section{An Analysis of Current Forms of Collaboration in SOMNet}

SOMNet aims to act as a hub and filter for knowledge in oral medicine, by providing a forum where cases in oral medicine can be presented and discussed in distributed meetings. SOMNet meetings are organized around monthly teleconferences, where about ten clinics participate. The majority of the attendants are experts in oral medicine and the individual clinician has the opportunity to get feedback from colleagues from all over Sweden. Mainly two kinds of cases are presented: cases considered interesting for a wider audience and cases where the submitting clinician wants advice regarding diagnosis and treatment.

The forms of cooperation and opinions of SOMNet have been investigated by observing their meetings and through informal interviews with selected participants and the secretary who coordinates the meetings. Before the SOMWeb initiative, submission of cases was handled by e-mailing PowerPoint presentations to the meeting coordinator, who then e-mailed submitted cases to clinics intending to participate. During the teleconference meeting, each clinic opened their local copy of the file presenting the case. The clinician who had submitted the case presented the details of the case, after which the community discussed it. When a case had been presented and discussed, the file was closed and attendants opened their local copy of the next case. They repeated this process until all submitted cases had been discussed or the time allotted to the meeting was up. At the meetings, no collective written notes were taken, and there was no central repository of handled cases.

The described procedure had several major problems: (1) The opportunities for collective and individual learning were limited by the lack of shared written notes from the meetings. (2) It was difficult to go back and check previously discussed cases as there was no shared record of treated cases. (3) Relevant information may be lacking in the case presentation, as there was no agreed upon template of what had to be included. It was left entirely up to the submitting individual what text and image information to include. (4) There were recurring problems of cases that could not be opened by all attendees, due to differences in versions of the software and platform used to produce the presentations.

Learning from earlier meetings is thus hindered by the lack of access to and reminders of previous cases, and by difficulties in structured comparison of different cases. Even with these problems, the participating clinicians value the opportunity to share experiences with other experts in the field enough to keep participating in the meetings, both by sending in cases and joining for discussion.

The introduction of a common web-based repository was a first step towards improving the IT-maturity of the SOMNet meetings. In this repository, the presentations are stored as HTML documents rather than as PowerPoint presentations. 
The new practice makes administration simpler and provides a bank of difficult and interesting cases. Since December 2003, when the clinicians first started using the repository, eighty-four cases from seventeen SOMNet meetings have been added.

A web-based questionnaire was distributed to all SOMNet participants to further examine the needs a wishes of the users. The results indicated that the participants considered SOMNet valuable and wanted it to continue in a similar way: as a forum for the most part dedicated to the discussion of submitted cases. Around seventy-five percent of the respondents also said that they wanted to be able to have access to the collection of treated cases. Ability to share cases and treatment methods and papers directly with other clinicians was of interest to a majority of the respondents. When the questionnaire was discussed at a later meeting, participants agreed that it was important to introduce a system that would allow the community to in a more organized manner follow up previously discussed cases. They also emphasized the importance of means to search the database of cases.

\section{System Design}

This section begins with the requirements we have previously identified for an ontology in oral medicine. We then present design choices for the oral medicine community which have lead us to use Semantic Web technologies and to build our own system, rather than using an existing Content Management System.

In [7], we presented the following requirements for an ontology for oral medicine, based on experience with the MedView system and interviews with domain experts and developers:

- We need the possibility and ability to utilize external sources of knowledge.

- The relation between the conceptual models of fundamental clinical concepts in use, e.g., examination templates, lists of approved values for terms and groups of related terms and their corresponding concrete entities must be formally examined.

- Relations and interactions between different entities of the ontology must be captured, e.g., that a certain answer to a specific question in a given examination template triggers another question.

- A stronger typing of elements is needed.

- We need to be able to capture different kinds of meta-data, e.g., who is the creator of a specific examination template and what the purpose (scientific or clinical) is of the introduction of a specific examination template.

- The localization of data has to be addressed rigorously: How to provide different language-based versions of the defined concepts, definitions and terms?

- We need to differentiate between different 'views' of the underlying data, to be utilized for, e.g., information visualization and intelligent user interfaces.

Based on these requirements an ontology for examinations in oral medicine was designed in OWL. Individual examination instances are encoded in RDF. Given that the examinations are an important part of the oral medicine community, and that these have Semantic Web representations, using such technologies 
for other relevant community concepts seems natural. Based on this, along with the analysis given in Sec. 3 we have made the following design choices:

- The community should be constructed in cooperation with its users, foremost to adapt it to the users needs, but also as the development of the online community can be seen as part of a learning process in the SOMNet users.

- The examinations should have a central role in the community and be represented in RDF, motivations for which are given above.

- The examinations to be presented at meetings should be entered online, using forms created from user-defined examinations, represented in OWL.

- Community data should be stored in RDF, to allow interaction with e.g., examination data and hopefully other related online communities in medicine.

- Community data will be stored centrally, rather than distributed as in e.g., [13], as the member clinicians and clinics cannot be expected to have access to local hosting.

- Where possible, existing ontologies for representing community data should be reused.

- To allow integration with the existing tools of the project, Java technologies should be used in realizing the community.

- The constructed community should allow for browsing and visualizing cases, as well as query support and search.

- To adapt to the needs of the users, the community should have basic user modeling support, with the long term goal of providing a more intelligent user interface.

- Our plans to use intelligent user interfaces and to keep a high degree of user customizability of the web interface in order to increase the clinicians trust in the system [18], point away from using a CMS in favor of using a lower-level framework with greater flexibility and end-user control.

While using a CMS would have given us a framework enabling rapid development of some aspects of our system, other aspects, e.g., generating web-based case entry forms from user-defined specifications, would still have to be custom-made from scratch.

\section{Implementation}

This section describes how the community data is represented using Semantic Web technologies and how Java web technologies are used to create the community framework.

\subsection{Semantic Web Technologies}

One part of the SOMWeb project is the formalization of clinical concepts and processes in oral medicine. Taking the knowledge model of MedView as a starting point, initial ontologies for examinations in oral medicine has been constructed [7] using OWL. There is one ontology for describing examination 
templates and one for classes of values that the examination templates use. The examination instances constructed using the ontology are stored as separate RDF-files.

The description of examination templates is further divided so that one 'central' OWL-file describes aspects common to all MedView examinations. This describes general things: classes such as Examination, ExaminationCategory, and a property hasExaminationCategory for connecting an Examination instance to ExaminationCategory instances. We also have properties corresponding to the different input-types, such as MultipleExaminationProperty, and VASExaminationProperty. The reason for explicitly representing things that could be handled using cardinality constraints in OWL is to be able to use older MedView datahandling classes to handle examinations. Until those modules can be rewritten, we are using both cardinality constraints and the explicit properties such as SingleExaminationProperty.

All individual examination templates refer to this 'central' OWL-file. In SOMWeb the main templates used right now describes what to enter when first entering the case into the system, as well as templates for consultations from the teleconference meeting and for consultations held after the initial examination data was entered. Such an examination template OWL-file contains definitions of the categories that can or need to be included in an examination constructed from that template. Examples of subclasses of ExaminationCategory in current use are PatientData, GeneralAnamnesis, and MucosChangeAnamnesis. In each examination template we also describe properties associated with the template, such as hasAllergy, as subproperties of the properties described in the general examination description OWL-file, such as MultipleExaminationProperty. For each property, there are also properties pertaining to description and instructions, to be shown to the user. Any relevant cardinality constraints are described, as well as the ordering of the categories and the properties within the categories, using rdf : list. Properties are connected to an ExaminationCategory subclass and a value class using owl :allValuesFrom restrictions.

The value list ontology is very simple, right now it only contains classes, with no subhierarchies, and their instances. Thus, we have classes such as Allergy and Diagnosis, with instances such as peanutAllergy and hairyTongue, respectively. It can be argued that this means that two patients who have examinations that assert that hasDiagnosis hairyTongue have the same hairy tongue, and that it would be more correct to have a class HairyTongue with separate instances for all patients with a diagnosis of hairy tongue. However, it was decided that the added complexity of this approach was not compensated by its benefits.

There is currently no use of inference, and the ontologies are not very complex. The examination templates are constructed by a Java program that takes a template in the old XML format and outputs one in OWL. The value list ontology is created by reading the old text file of values and constructing corresponding classes and instances. At first there were attempts to structure this value list so that there were more subclasses, for example for different kinds of diagnoses and allergies. However, this process meant dealing with large amounts of instances 


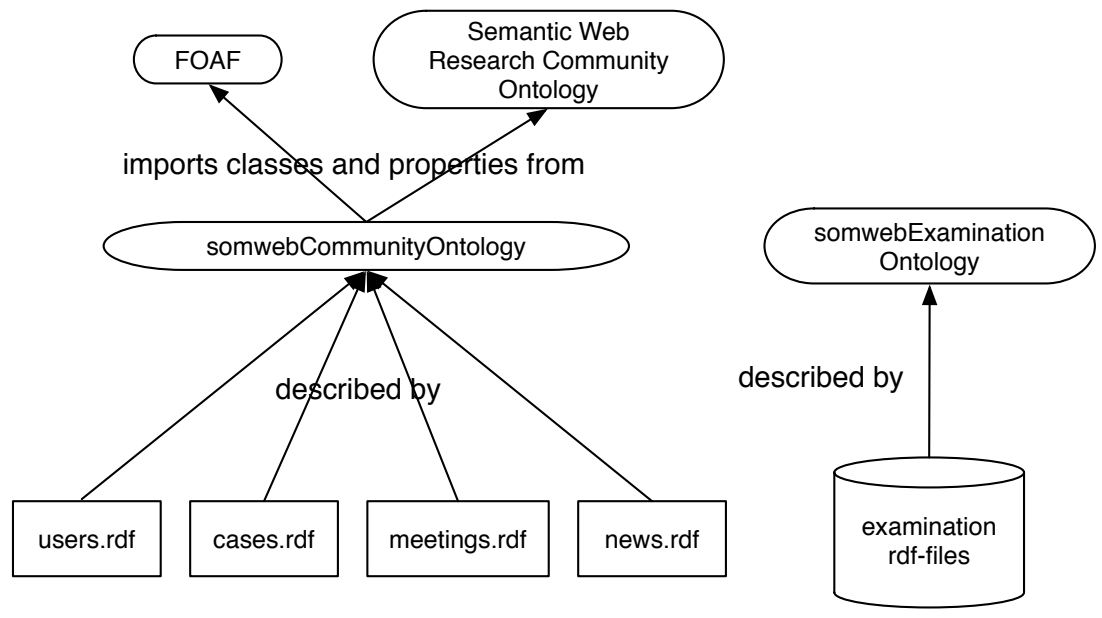

Fig. 1. The figure shows how the different RDF-files and OWL ontologies are related. The RDF-files for persons, meetings, cases (examination metadata), and news are described in the somwebCommunityOntology. The examinations are stored in separate RDF-files, and are described in the somwebExaminationOntology. The examination ontology consists of one OWL-file describing general concepts and then one for each kind of examination used in the community.

and needed much end-user input. Our end-users have not yet had the time to look at this, and we have no good interface for them to do it in. Therefore we decided to go ahead with the unstructured value classes, with the argument that we can add more subclasses later.

In addition to storing the examinations in RDF, other community data, regarding users, meetings, news, and case metadata, is also represented in RDF. What should be included in descriptions of users, meetings, and case metadata is described in OWL. Some of the user-descriptions are related to relevant FOAFclasses and properties. Meeting-descriptions make use of classes and properties of the Semantic Web Research Community ontology [17]. For interacting with the Java parts of the community, Jena ${ }^{6}$ is used. Figure 1 shows how these components are related.

\subsection{Java Web Technologies}

SOMWeb is built on Java Enterprise technology, using Apache Tomcat 7 as the core web container. The system is an extension of the Apache Struts Model-2 web application framework 8 . Model-2 frameworks (a variation of the classic ModelView-Controller (MVC) design paradigm) are based on the idea that Java Servlets execute business logic while presentation resides mainly in server pages. As stated

\footnotetext{
6 http://jena.sourceforge.net/

7 http://tomcat .apache.org

8 http://struts.apache.org/
} 


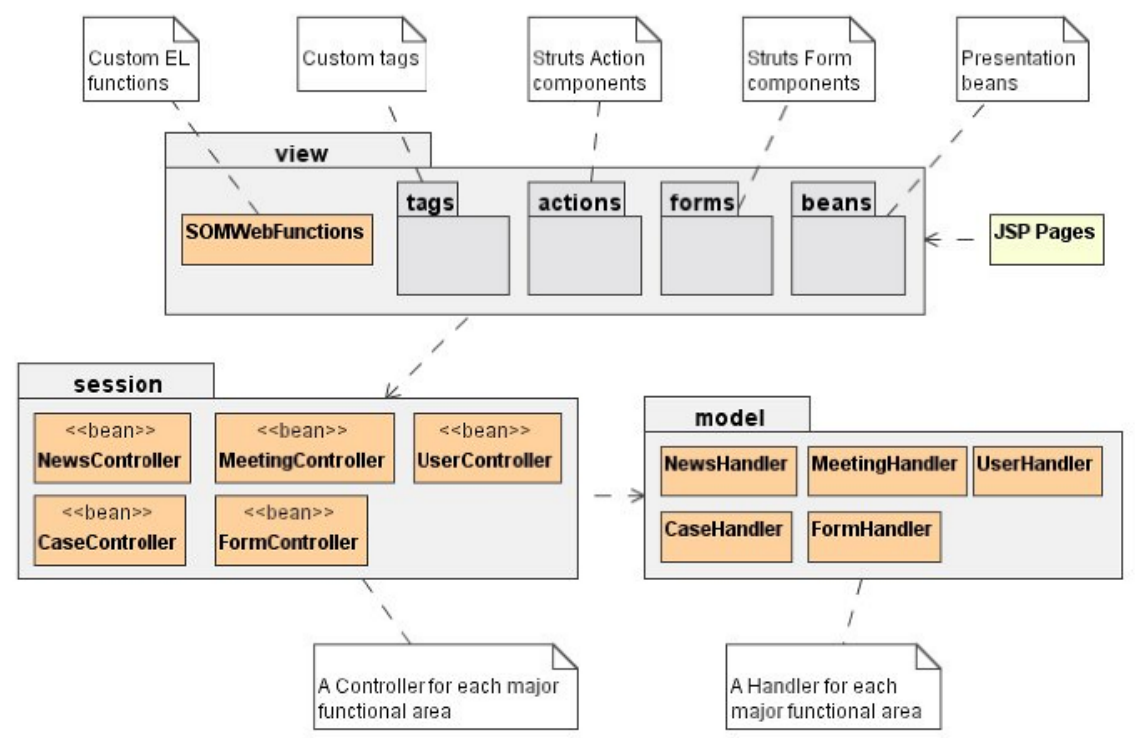

Fig. 2. Overview of the SOMWeb system architecture. The model layer contains persistence classes that read RDF-files for users, meetings, cases, and news and constructs objects of the corresponding Java classes used by the system. These classes are also used for making changes to the RDF model and writing to file.

in [12, reusing mature technology gives a Semantic Web portal improved usability, reliability, and scalability.

The SOMWeb system is a layered architecture, conceptually divided into four main layers - the view layer, the session layer, the model layer, and the foundation layer as depicted in Fig. 2. The view layer is comprised of Java Server Pages (JSP) using Expression Language (EL) constructs, with custom tags and functions in addition to tags from the Java Standards Tag Library (JSTL) and the various Apache Struts tag libraries. Styling and layout of content is done using Cascading Style Sheets (CSS). The session layer has components dealing with the current user session, and is responsible for transforming the application's internal state into the presentation JavaBeans used by the server pages. The model layer has components making up the application's internal state, and is roughly divided into the major functional areas provided by the system. Here we also have persistence classes, which read the RDF-files for users, meetings, cases, and news and creates objects of the corresponding Java-classes used by the system. These persistence classes are manually constructed, but in the future we are considering using Jastor 9 to simplify their updating. While the generation of user input forms from OWL is handled by the SOMWeb system, for handling examination data we use the previously developed MedView system and its datahandling functions, which have been adapted to using OWL and RDF.

9 http://jastor.sourceforge.net/ 


\section{Results}

The initial SOMWeb community supports case data entry according to userdefined templates, managing online meetings and assigning cases to these, viewing upcoming and archived meetings and their corresponding cases, and adding comments to cases. Users can use free text search over examination data. Administrators are provided basic user handling, create an manage meetings, and post news.

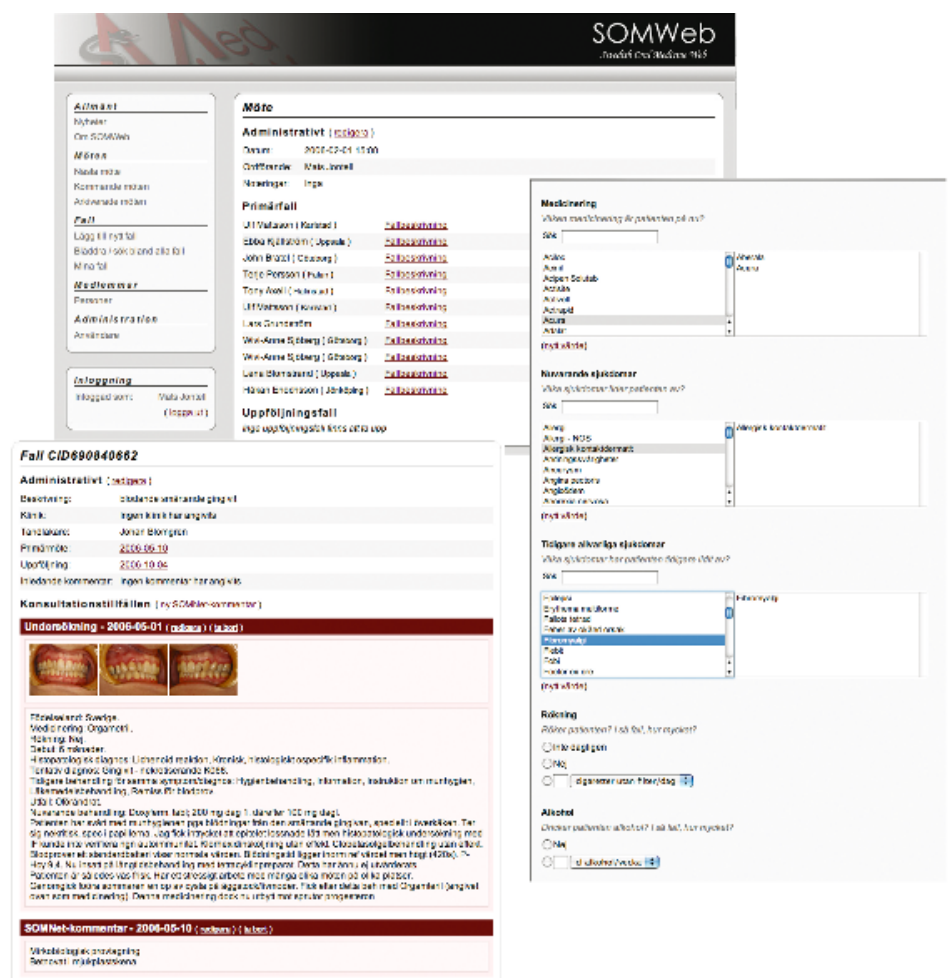

Fig. 3. The figure shows screenshots of some key parts of SOMWeb: overview of cases at a meeting (top), case presentation with pictures and text description generated from examination data (left), and part of an examination data entry form (right). All text is in Swedish.

Figure 3 shows screenshots of core system aspects, such as structured case entry - the user is presented with a blank form based on a user-defined OWL description of the examination, in which the user enters case data. The examination description is created in a separate editor for specifying the content of the examination, and the user never interacts with the OWL representation. The types of questions posed and the allowed values are determined by the underlying OWL description. After 
submission, the case data is stored as RDF and a summary of the case information can be presented (also shown in Fig. 3).

Shown in the case presentation is administrative information along with a system-generated medical record. The record is constructed from the submitted case data and generated by the mGen document generator, using natural language processing technology, as described in [19]. If more information about a case becomes available, the owner can use forms specifically tailored for this purpose to add new data. Each such data occasion is reflected in the case summary and a record is generated for each occasion. The owner of a case can also schedule the case for initial discussion at a SOMNet meeting, as well as selecting a meeting at which the case should be considered again, for possible follow-up and re-evaluation.

\section{Discussion}

While the ontologies used in the SOMWeb community are fairly simple, we believe that it is still motivated to use OWL and RDF in our application, both because it, among other things, gives us access to tools for interacting with the $\mathrm{RDF}$ content and because we can return in the future and refine our ontology. In the old MedView representation there was no support for refining the knowledge model by subclassing the value classes, and a separate 'aggregration' representation was used for this. When instead using OWL, this can be achieved using one representation. Indeed, Knublauch et al. 20] argue that "the breadth of the OWL language offers a migration route from entry level, hand-crafted taxonomies of terms, to well defined, normalized ontologies capable of supporting reasoning." Thus, at first a semantically simple ontologies such as these is enough to make a Web application able to generate user interface forms from class definitions and describe schema useful for integration. Then, later in the ontology's life cycle, additional expressivity can be added as developers find they need it.

However, OWL and RDF are still fairly young technologies, with the effect that developers are not as familiar with it as with for example regular XML, which means that initially more time is spent getting familiar with new nomenclature and new API's. In designing the larger examination template ontologies, the initial barriers were larger than for the community data ontology. We were faced with confusion in the usage of domain and range, as well as a lack of support for representing for example numeric ranges. Also, for the examination template ontology there was more of a feeling that the 'right' choices had to be made, which sometimes lead to stalled progress.

SOMWeb has been designed to support the collaborative work in SOMNet (and similar organisations), especially the tasks of setting up and holding meetings, from the perspectives of the different types of users. Focusing on behavioural aspects of clinical research - modelling and implementing the 'workflows' of SOMNet, makes SOMWeb more of a workflow management system [21] than a traditional CMS.

While this project has the goal of constructing an online community, it was found that what the clinicians wanted was not so much to discuss the cases 
online, but to have a common memory there, where they could contribute and find cases. The informal communication was less sought after, and was taken care of in teleconferences and in person or by phone. A drawback of this is that no trail is left after these conversations, which might have benefited those not present.

\section{Conclusions and Future Work}

We have shown how Semantic Web technologies can be used to support online collaborative work in a small medical speciality. The SOMWeb online community uses RDF for representing central components such as medical examinations and case metadata, community users, scheduled teleconference meetings, and news messages. Where relevant, classes and properties from the FOAF and SWRC ontologies have been reused. These RDF representations interact with the larger Apache Struts framework through the Jena API.

By using RDF for representing the underlying information of SOMWeb we hope to gain increased interoperability, and the ability to use SPARQL. Future work includes allowing users to pose structured queries on the collected data, rather than using free text search, allowing precise answers rather than pieces of documents that match the keyword. However, the users of SOMWeb have little knowledge that the underlying representation of their online community is RDF, and there should not be a need for them knowing this. Therefore, the structured queries need to be characterized in a manner graspable without understanding a more complex underlying design.

There is currently no support in the community for informal communication, through for example online forums, but such features will soon be provided and eventually be compatible with SIOC. While the information of SOMWeb is only available by password identification, we believe that using RDF is still very useful, since we get the possiblity of reusing concepts from larger community ontologies such SIOC. An enticing possibility is to connect several related medical online communities of practice through SPARQL web services.

\section{References}

1. Jim Q. Chen, J.H., Ted E. Lee, J.H., Zhang, R.: Systems requirements for organizational learning. Communications of the ACM 46(12) (2003) 73-79

2. Bose, R.: Knowledge management-enabled health care management systems: capabilities, infrastructure, and decision-support. Expert Systems with Applications 24(1) (2003) 59-71

3. Dawes, M., Sampson, U.: Knowledge management in clinical practice: a systematic review of information seeking behavior in physicians. International Journal of Medical Informatics 71(1) (2003) 9-15

4. Ryu, S., Ho, S., Han, I.: Knowledge sharing behavior of physicians in hospitals. Expert Systems with Applications 25(1) (2003) 113-122

5. Falkman, G., Torgersson, O., Jontell, M., Gustafsson, M.: SOMWeb - Towards an infrastructure for knowledge sharing. In: Proc. Medical Informatics Europe, IOS Press (2005) 527-32 
6. Jontell, M., Mattsson, U., Torgersson, O.: MedView: An instrument for clinical research and education in oral medicine. Oral Surg. Oral Med. Oral Pathol. Oral Radiol. Endod. 99 (2005) 55-63

7. Gustafsson, M., Falkman, G.: Representing clinical knowledge in oral medicine using ontologies. In: Proc. Medical Informatics Europe, IOS Press (2005) 743-8

8. Gustafsson, M., Lindahl, F., Falkman, G., Torgersson, O.: An online community for oral medicine supporting structured case entry. In: Poster and Demonstration Proc. MIE 2006. (2006) 469-474

9. Neumann, E.K., Miller, E., Wilbanks, J.: What the semantic web could do for the life sciences. Drug Discovery Today BioSilico 2(6) (2004) 228-236

10. Lambrix, P.: Towards a semanticweb for bioinformatics using ontology-based annotation. In: WETICE '05: Proceedings of the 14th IEEE International Workshops on Enabling Technologies: Infrastructure for Collaborative Enterprise, Washington, DC, USA, IEEE Computer Society (2005) 3-7

11. Heflin, J.: Web Ontology Language (OWL): use cases and requirements. W3C Recommendation 10 February 2004 (2004)

12. Lausen, H., Ding, Y., Stollberg, M., Fensel, D., Hernandez, R.L., Han, S.K.: Semantic web portals: state-of-the-art survey. Journal of Knowledge Management 9(5) (2005) 40-49

13. Reynolds, D., Shabajee, P., Cayzer, S., Steer, D.: SWAD-Europe deliverable 12.1.7: Semantic portals demonstrator - lessons learnt (2004)

14. Breslin, J.G., Harth, A., Bojars, U., Decker, S.: Towards semantically-interlinked online communities. In Gómez-Pérez, A., Euzenat, J., eds.: The Semantic Web: Research and Applications, Second European Semantic Web Conference, ESWC 2005, Heraklion, Crete, Greece, May 29 - June 1, 2005, Proceedings. (2005) 500-514

15. Stojanovic, N., Maedche, A., Staab, S., Studer, R., Sure, Y.: SEAL - A framework for developing SEmantic portALs. In: K-CAP 2001 - First Intenational Conference on Knowledge Capture, Victoria, Canada, Oct. 21-23, 2001, ACM (2001)

16. Decker, S., Erdmann, M., Fensel, D., Studer, R.: Ontobroker: Ontology based access to distributed and semi-structured information. In Meersman, R., ed.: Database Semantics: Semantic Issues in Multimedia Systems, Proceedings TC2/WG 2.6 8th Working Conference on Database Semantics (DS-8), Rotorua, New Zealand, Kluwer Academic Publishers, Boston (1999)

17. Sure, Y., Bloehdorn, S., Haase, P., Hartmann, J., Oberle, D.: The SWRC ontology - Semantic Web for research communities. In Bento, C., Cardoso, A., Dias, G., eds.: Proceedings of the 12th Portuguese Conference on Artificial Intelligence Progress in Artificial Intelligence (EPIA 2005). Volume 3803 of LNCS., Covilha, Portugal, Springer (2005) 218-231

18. Wetter, T.: Lessons learnt from bringing knowledge-based systems into routine use. Artif. Intell. Med. 24(3) (2002) 195-203

19. Lindahl, F., Torgersson, O.: mGen - An open source framework for generating clinical documents. In: Proc. Medical Informatics Europe, IOS Press (2005) 107-12

20. Knublauch, H., Horridge, M., Musen, M., Rector, A., Stevens, R., Drummond, N., Lord, P., Noy, N.F., Seidenberg, J., Wang, H.: The Protgé OWL Experience. In: Proc. of the Workshop on OWL Experiences and Directions 2005, Galway, Ireland (2005)

21. Pratt, W., Reddy, M., McDonald, D., Tarczy-Hornoch, P., Gennari, J.: Incorporating ideas from computer-supported cooperative work. Journal of Biomedical Informatics 37(2) (2004) 128-137 